\title{
PENERAPAN METODE BESARAN PIVOT DALAM PENURUNAN RUMUS TAKSIRAN INTERVAL DARI KOEFISIEN REGRESI LINEAR SEDERHANA
}

Oleh :

\author{
Nar Herrhyanto \\ Jurusan Pendidikan Matematika, FPMIPA, UPI
}

\begin{abstract}
Abstrak
Regresi merupakan bentuk hubungan antara variabel bebas $\mathrm{X}$ dan variabel respon $\mathrm{Y}$ yang dinyatakan dalam sebuah persamaan matematik. Persamaan matematik tsb selanjutnya akan merupakan sebuah persamaan regresi. Persamaan regresi linear yang sebenarnya berbentuk $Y=\alpha+\beta X+\varepsilon$. Dari koefisien regresi $\alpha$ dan $\beta$, maka $\beta$ merupakan koefisien regresi yang berpengaruh terhadap perubahan variabel respon $Y$.

Karena nilai $\beta$ ini biasanya tidak diketahui, maka nilainya akan ditaksir berdasarkan data sampel. Dalam hal ini, penaksiran $\beta$ yang akan dibahas dalam tulisan ini adalah penaksiran interval. Dengan kata lain, bagaimana bentuk rumus taksiran interval dari $\beta$ ini. Sehingga dalam makalah ini akan dijelaskan bagaimana penurunan rumus taksiran interval dari $\beta$. Dalam statistika matematik ada sebuah metode yang digunakan dalam penaksiran interval ini, yaitu metode besaran pivot.
\end{abstract}

Kata Kunci : Regresi Linear Sederhana, Taksiran Interval Koefisien Regresi, Besaran Pivot

\section{Pendahuluan}

Dalam kehidupan sehari-hari banyak data yang diperoleh atau dikumpulkan dengan melibatkan variabel. Apabila ada dua variabel, yaitu variabel bebas dan variabel respon, maka dapat diketahui hubungan antara kedua variabel tsb. Variabel bebas adalah variabel yang nilainya sudah diketahui dan biasanya dinyatakan dengan X. Variabel respon adalah variabel yang nilainya ditentukan berdasarkan variabel bebas, dan biasanya dinyatakan dengan Y. Dari kedua variabel tsb, dapat diketahui bentuk hubungan antara keduanya yang dinyatakan dengan sebuah persamaan matematik. Bentuk hubungan tsb sering disebut sebagai regresi. 
Regresi ada dua macam, yaitu regresi linear dan regresi non linear. Penentuan regresi linear atau non linear ini bisa diketahui melalui metode tangan bebas. Dalam metode tangan bebas ini didasarkan pada diagram pencar, yaitu diagram yang dibentuk berdasarkan data berpasangan $(\mathrm{x}, \mathrm{y})$ dan berupa sekumpulan titik-titk yang terpencar dimana-mana. Apabila letak sekumpulan titik-titik itu sekitar garis lurus, maka regresi yang diperoleh berbentuk linear. Apabila letak sekumpulan titik-titik itu membentuk sebuah kurva, maka regresi yang diperoleh berbentuk non linear. Dalam hal ini akan dibahas regresi yang berbentuk linear saja. Regresi linearnya juga merupakan regresi linear sederhana, yaitu regresi yang melibatkan satu variabel bebas $\mathrm{X}$ dan satu variabel respon Y.

Persamaan regresi yang sebenarnya berbentuk $Y=\alpha+\beta X+\varepsilon$. Dari koefisien regresi $\alpha$ dan $\beta$, maka $\beta$ merupakan koefisien regresi yang berpengaruh terhadap perubahan variabel respon Y. Artinya $\beta$ merupakan koefisien yang menyatakan perubahan variabel respon $\mathrm{Y}$, apabila variabel bebas X berubah sebesar satu satuan. Karena nilai $\beta$ ini biasanya tidak diketahui, maka nilainya akan ditaksir berdasarkan data sampel.

Dalam statistika dibahas penyelesaian suatu masalah, mulai dari pengumpulan data sampai penarikan kesimpulan. Salah satu cara yang digunakan untuk menarik kesimpulan adalah berhubungan dengan penaksiran parameter. Penaksiran parameter adalah penentuan sebuah nilai atau nilai-nilai yang dihitung dari sampel acak sebagai pengganti dari sebuah parameter yang nilainya tidak diketahui. Penentuan nilai dari sampel acak ini disesuaikan dengan parameternya. Penaksiran interval merupakan cara untuk menentukan nilai-nilai yang berbentuk interval berdasarkan data sampel, dimana nilai-nilai tsb sebagai pengganti dari nilai parameter yang tidak diketahui. Dalam penentuan taksiran interval tsb harus dicari dahulu taksiran titik dari parameter yang bersesuaiannya. Dalam statistika yang membahas rumusrumusnya secara teoritis (disebut statistika matematik) ada sebuah metode yang digunakan untuk menentukan taksiran interval dari sebuah parameter, yaitu metode besaran pivot.

\section{Permasalahan}

Dalam statistika ada dua cara untuk mempelajarinya, yaitu menggunakan rumus-rumusnya dan menurunkan rumus-rumusnya. Orang yang mempelajari statistika, selain menggunakan rumus yang ada sebaiknya harus dipelajari juga bagaimana rumus-rumus itu diturunkan. Untuk menurunkan rumus dalam statistika diperlukan materi prasyarat, sehingga masalah ini merupakan masalah teoritis. Sehubungan dengan masalah teoritis ini, maka penulis 
berusaha untuk menurunkan rumus-rumus yang terdapat dalam statistika khususnya dalam regresi. Salah satu masalah yang terdapat dalam regresi adalah taksiran interval dari koefisien regresi dalam regresi linear sederhana. Sehingga permasalahan yang akan dibahas dalam tulisan ini adalah bagaimana penurunan rumus taksiran interval untuk koefisien regresi linear sederhana dengan menggunakan metode besaran pivot?

\section{Pembahasan}

Menurut Herrhyanto (2003:156) dan (2010:52), langkah-langkah yang diperlukan untuk menentukan taksiran interval sebuah parameter dari sebuah distribusi dengan menggunakan metode besaran pivot sbb:

1. Tentukan taksiran titik dari parameter itu. Dalam hal ini, tentunya dicari penaksir tak bias bagi parameter itu.

2. Tentukan distribusi dari penaksir tak bias itu (kalau diperlukan).

3. Tentukan besaran pivot, yaitu besaran yang mengandung penaksir dan parameter sedemikian hingga distribusinya tidak bergantung pada parameternya.

4. Tentukan distribusi dari besaran pivot.

5. Besaran pivot itu disubstitusikan kedalam bentuk umum dari taksiran interval dengan derajat keyakinan sebesar $(1-\alpha)$, yaitu :

$$
\mathrm{P}(\mathrm{a}<\text { besaran pivot }<\mathrm{b})=1-\alpha
$$

6. Ubah bentuk dalam langkah kelima kedalam bentuk:

$$
\mathrm{P}(\mathrm{c}<\text { parameter }<\mathrm{d})=1-\alpha
$$

Berikut ini akan dijelaskan langkah-langkah di atas.

\section{Penaksir Titik Parameter yang Tak Bias}

Persamaan regresi linear sederhana mempunyai bentuk $Y=\alpha+\beta X+\varepsilon$ dan persamaan regresi taksirannya berbentuk $\hat{Y}=\hat{\alpha}+\hat{\beta} X$. Karena Y berdistribusi $\mathrm{N}\left(\alpha+\beta \mathrm{x} ; \sigma^{2}\right)$, maka fungsi kepadatan peluang dari $Y$ berbentuk:

$$
f(y)=\frac{1}{\sqrt{2 \pi \cdot \sigma^{2}}} \exp \left[\frac{-1}{2 \sigma^{2}}(y-\alpha-\beta x)^{2}\right] ;-\infty<y<\infty
$$

Dalam hal ini akan ditentukan penaksir dari $\alpha$ dan $\beta$ dengan menggunakan metode kemungkinan maksimum.

Fungsi kemungkinan dari $\mathrm{Y}_{1}, \mathrm{Y}_{2}, \ldots, \mathrm{Y}_{\mathrm{n}}$ adalah: 


$$
\begin{aligned}
& \mathrm{L}\left(\alpha, \beta, \sigma^{2} ; \mathrm{y}_{1}, \mathrm{y}_{2}, \ldots, \mathrm{y}_{\mathrm{n}}\right)=\frac{1}{\sqrt{2 \pi \cdot \sigma^{2}}} \exp \left[\frac{-1}{2 \sigma^{2}}\left(y_{1}-\alpha-\beta x_{1}\right)^{2}\right] \\
& \mathrm{x} \frac{1}{\sqrt{2 \pi \cdot \sigma^{2}}} \exp \left[\frac{-1}{2 \sigma^{2}}\left(y_{2}-\alpha-\beta x_{2}\right)^{2}\right] \\
& \mathrm{x} \ldots \mathrm{x} \frac{1}{\sqrt{2 \pi \cdot \sigma^{2}}} \exp \left[\frac{-1}{2 \sigma^{2}}\left(y_{n}-\alpha-\beta x_{n}\right)^{2}\right] \\
&=\left(\frac{1}{\sqrt{2 \pi \cdot \sigma^{2}}}\right)^{n} \exp \left[\frac{-1}{2 \sigma^{2}} \sum_{i=1}^{n}\left(y_{i}-\alpha-\beta x_{i}\right)^{2}\right] \\
& \ln \mathrm{L}\left(\alpha, \beta, \sigma^{2} ; \mathrm{y}_{1}, \mathrm{y}_{2}, \ldots, \mathrm{y}_{\mathrm{n}}\right)=n \cdot \ln \left(\frac{1}{\sqrt{2 \pi \cdot \sigma^{2}}}\right)-\frac{1}{2 \sigma^{2}} \sum_{i=1}^{n}\left(y_{i}-\alpha-\beta x_{i}\right)^{2} \\
& \frac{\partial}{\partial \alpha} \ln L\left(\alpha, \beta, \sigma^{2} ; y_{1}, y_{2}, \ldots, y_{n}\right)=-\frac{1}{2 \sigma^{2}} \cdot 2 \cdot \sum_{i=1}^{n}\left(y_{i}-\alpha-\beta x_{i}\right)(-1) \\
&=\frac{1}{\sigma^{2}} \sum_{i=1}^{n}\left(y_{i}-\alpha-\beta x_{i}\right) \\
& \begin{array}{r}
\frac{\partial}{\partial \beta} \ln L\left(\alpha, \beta, \sigma^{2} ; y_{1}, y_{2}, \ldots, y_{n}\right)=-\frac{1}{2 \sigma^{2}} \cdot 2 \cdot \sum_{i=1}^{n}\left(y_{i}-\alpha-\beta x_{i}\right)\left(-x_{i}\right) \\
=\frac{1}{\sigma^{2}} \sum_{i=1}^{n}\left(y_{i}-\alpha-\beta x_{i}\right)\left(x_{i}\right)
\end{array}
\end{aligned}
$$

\section{Syarat :}

$$
\begin{array}{ll}
\text { a. } & \frac{\partial}{\partial \alpha} \ln L\left(\alpha, \beta, \sigma^{2} ; y_{1}, y_{2}, \ldots, y_{n}\right)=0 \\
& \frac{1}{\sigma^{2}} \sum_{i=1}^{n}\left(y_{i}-\hat{\alpha}-\hat{\beta} x_{i}\right)=0 \\
\sum_{i=1}^{n}\left(y_{i}-\hat{\alpha}-\hat{\beta} x_{i}\right)=0 \\
\sum_{i=1}^{n} y_{i}-n \cdot \hat{\alpha}-\hat{\beta} \cdot \sum_{i=1}^{n} x_{i}=0 \\
\sum_{i=1}^{n} y_{i}=n \cdot \hat{\alpha}+\hat{\beta} \cdot \sum_{i=1}^{n} x_{i}
\end{array}
$$


b. $\frac{\partial}{\partial \beta} \ln L\left(\alpha, \beta, \sigma^{2} ; y_{1}, y_{2}, \ldots, y_{n}\right)=0$

$$
\begin{aligned}
& \frac{1}{\sigma^{2}} \sum_{i=1}^{n}\left(y_{i}-\hat{\alpha}-\hat{\beta} x_{i}\right)\left(x_{i}\right)=0 \\
& \sum_{i=1}^{n}\left(y_{i}-\hat{\alpha}-\hat{\beta} x_{i}\right)\left(x_{i}\right)=0 \\
& \sum_{i=1}^{n} x_{i} y_{i}-\hat{\alpha} \cdot \sum_{i=1}^{n} x_{i}-\hat{\beta} \cdot \sum_{i=1}^{n} x_{i}^{2}=0 \\
& \sum_{i=1}^{n} x_{i} y_{i}=\hat{\alpha} \cdot \sum_{i=1}^{n} x_{i}+\hat{\beta} \cdot \sum_{i=1}^{n} x_{i}^{2}
\end{aligned}
$$

Jadi diperoleh dua persamaan normal, yaitu:

- $\sum_{i=1}^{n} y_{i}=n . \hat{\alpha}+\hat{\beta} \cdot \sum_{i=1}^{n} x_{i}$

- $\quad \sum_{i=1}^{n} x_{i} y_{i}=\hat{\alpha} \cdot \sum_{i=1}^{n} x_{i}+\hat{\beta} \cdot \sum_{i=1}^{n} x_{i}^{2}$

Dengan menggunakan Aturan Cramer diperoleh :

$$
\begin{aligned}
& \hat{\alpha}=\frac{\left(\sum_{i=1}^{n} y_{i}\right)\left(\sum_{i=1}^{n} x_{i}^{2}\right)-\left(\sum_{i=1}^{n} x_{i}\right)\left(\sum_{i=1}^{n} x_{i} y_{i}\right)}{n \cdot \sum_{i=1}^{n} x_{i}^{2}-\left(\sum_{i=1}^{n} x_{i}\right)^{2}} \\
& \hat{\beta}=\frac{n \cdot\left(\sum_{i=1}^{n} x_{i} y_{i}\right)-\left(\sum_{i=1}^{n} x_{i}\right)\left(\sum_{i=1}^{n} y_{i}\right)}{n \cdot \sum_{i=1}^{n} x_{i}^{2}-\left(\sum_{i=1}^{n} x_{i}\right)^{2}}
\end{aligned}
$$

Jadi penaksir kemungkinan maksimum bagi $\alpha$ dan $\beta$ adalah:

$$
\begin{gathered}
\hat{\alpha}=\frac{\left(\sum_{i=1}^{n} Y_{i}\right)\left(\sum_{i=1}^{n} X_{i}^{2}\right)-\left(\sum_{i=1}^{n} X_{i}\right)\left(\sum_{i=1}^{n} X_{i} Y_{i}\right)}{n \cdot \sum_{i=1}^{n} X_{i}^{2}-\left(\sum_{i=1}^{n} X_{i}\right)^{2}} \\
\hat{\beta}=\frac{n \cdot\left(\sum_{i=1}^{n} X_{i} Y_{i}\right)-\left(\sum_{i=1}^{n} X_{i}\right)\left(\sum_{i=1}^{n} Y_{i}\right)}{n \cdot \sum_{i=1}^{n} X_{i}^{2}-\left(\sum_{i=1}^{n} X_{i}\right)^{2}}
\end{gathered}
$$


Atau bisa juga diperoleh:

$$
\begin{aligned}
& \sum_{i=1}^{n} y_{i}=n \cdot \hat{\alpha}+\hat{\beta} \cdot \sum_{i=1}^{n} x_{i} \\
& \frac{1}{n} \sum_{i=1}^{n} y_{i}=\hat{\alpha}+\hat{\beta} \cdot \frac{1}{n} \sum_{i=1}^{n} x_{i} \\
& \bar{y}=\hat{\alpha}+\hat{\beta} \cdot \bar{x} \\
& \hat{\alpha}=\bar{y}-\hat{\beta} \cdot \bar{x}
\end{aligned}
$$

Jadi penaksir bagi $\alpha$ berbentuk: $\hat{\alpha}=\bar{Y}-\hat{\beta} \cdot \bar{X}$

Dan penaksir $\hat{\beta}$ nya bisa ditulis sbb:

$$
\begin{aligned}
& \hat{\beta}=\frac{n \cdot\left(\sum_{i=1}^{n} X_{i} Y_{i}\right)-\left(\sum_{i=1}^{n} X_{i}\right)\left(\sum_{i=1}^{n} Y_{i}\right)}{n \cdot \sum_{i=1}^{n} X_{i}^{2}-\left(\sum_{i=1}^{n} X_{i}\right)^{2}} \\
& \hat{\beta}=\frac{\sum_{i=1}^{n} X_{i} Y_{i}-n \cdot \bar{X} \cdot \bar{Y}}{\sum_{i=1}^{n} X_{i}^{2}-n \cdot \bar{X}^{2}}
\end{aligned}
$$

Kemudian akan diperiksa bahwa $\hat{\beta}$ harus merupakan penaksir tak bias bagi $\beta$. Dari penaksir $\hat{\beta}$ akan diuraikan bentuk pada pembilang maupun penyebutnya satu persatu.

$$
\begin{aligned}
\sum_{i=1}^{n} X_{i} Y_{i}-n \cdot \bar{X} \cdot \bar{Y} & =\sum_{i=1}^{n} X_{i} Y_{i}-\bar{X} \cdot \sum_{i=1}^{n} Y_{i} \\
& =\sum_{i=1}^{n}\left(X_{i} Y_{i}-\bar{X} \cdot Y_{i}\right) \\
& =\sum_{i=1}^{n}\left(X_{i}-\bar{X}\right) . Y_{i}
\end{aligned}
$$




$$
\begin{aligned}
& \sum_{i=1}^{n} X_{i}^{2}-n \cdot \bar{X}^{2}=\sum_{i=1}^{n} X_{i}^{2}-2 \cdot n \cdot \bar{X}^{2}+n \cdot \bar{X}^{2} \\
&=\sum_{i=1}^{n} X_{i}^{2}-2 \cdot \bar{X} \cdot \sum_{i=1}^{n} X_{i}+n \cdot \bar{X}^{2} \\
&=\sum_{i=1}^{n}\left(X_{i}^{2}-2 \cdot \bar{X} \cdot X_{i}+\bar{X}^{2}\right) \\
&=\sum_{i=1}^{n}\left(X_{i}-\bar{X}\right)^{2}
\end{aligned}
$$

Sehingga:

$$
\begin{aligned}
& \hat{\beta}=\frac{\sum_{i=1}^{n}\left(X_{i}-\bar{X}\right) Y_{i}}{\sum_{i=1}^{n}\left(X_{i}-\bar{X}\right)^{2}} \\
& \hat{\beta}=\frac{\left(X_{1}-\bar{X}\right) Y_{1}}{\sum_{i=1}^{n}\left(X_{i}-\bar{X}\right)^{2}}+\frac{\left(X_{2}-\bar{X}\right) Y_{2}}{\sum_{i=1}^{n}\left(X_{i}-\bar{X}\right)^{2}}+\ldots+\frac{\left(X_{n}-\bar{X}\right) Y_{n}}{\sum_{i=1}^{n}\left(X_{i}-\bar{X}\right)^{2}} \\
& \hat{\beta}=c_{1} Y_{1}+c_{2} Y_{2}+\ldots+c_{n} Y_{n}
\end{aligned}
$$

Dengan : (i) $\quad c_{1}=\frac{\left(X_{1}-\bar{X}\right)}{\sum_{i=1}^{n}\left(X_{i}-\bar{X}\right)^{2}}$

$$
\begin{aligned}
& \text { (ii) } c_{2}=\frac{\left(X_{2}-\bar{X}\right)}{\sum_{i=1}^{n}\left(X_{i}-\bar{X}\right)^{2}} \\
& \text { (iii) } c_{n}=\frac{\left(X_{n}-\bar{X}\right)}{\sum_{i=1}^{n}\left(X_{i}-\bar{X}\right)^{2}}
\end{aligned}
$$

Karena $Y_{1}, Y_{2}, \ldots, Y_{n}$ adalah sampel acak berukuran $n$ yang berasal dari populasi berdistribusi $\mathrm{N}\left(\alpha+\beta \mathrm{X}_{\mathrm{i}} ; \sigma^{2}\right)$, maka:

$$
\begin{aligned}
E(\hat{\beta}) & =E\left(c_{1} Y_{1}+c_{2} Y_{2}+\ldots+c_{n} Y_{n}\right) \\
& =E\left(c_{1} Y_{1}\right)+E\left(c_{2} Y_{2}\right)+\ldots+E\left(c_{n} Y_{n}\right) \\
& =c_{1} \cdot E\left(Y_{1}\right)+c_{2} \cdot E\left(Y_{2}\right)+\ldots+c_{n} \cdot E\left(Y_{n}\right)
\end{aligned}
$$




$$
\begin{aligned}
& =c_{1}\left(\alpha+\beta X_{1}\right)+c_{2}\left(\alpha+\beta X_{2}\right)+\ldots+c_{n}\left(\alpha+\beta X_{n}\right) \\
& =\alpha\left(c_{1}+c_{2}+\ldots+c_{n}\right)+\beta\left(c_{1} X_{1}+c_{2} X_{2}+\ldots+c_{n} X_{n}\right)
\end{aligned}
$$

\section{Dengan :}

$$
\begin{aligned}
\mathrm{c}_{1}+\mathrm{c}_{2}+\ldots+\mathrm{c}_{\mathrm{n}} & =\frac{\left(X_{1}-\bar{X}\right)}{\sum_{i=1}^{n}\left(X_{i}-\bar{X}\right)^{2}}+\frac{\left(X_{2}-\bar{X}\right)}{\sum_{i=1}^{n}\left(X_{i}-\bar{X}\right)^{2}}+\ldots+\frac{\left(X_{n}-\bar{X}\right)}{\sum_{i=1}^{n}\left(X_{i}-\bar{X}\right)^{2}} \\
& =\frac{\sum_{i=1}^{n}\left(X_{i}-\bar{X}\right)}{\sum_{i=1}^{n}\left(X_{i}-\bar{X}\right)^{2}} \\
& =\frac{0}{\sum_{i=1}^{n}\left(X_{i}-\bar{X}\right)^{2}} \\
\mathrm{c}_{1}+\mathrm{c}_{2}+\ldots+\mathrm{c}_{\mathrm{n}} & =0
\end{aligned}
$$

- $\mathrm{c}_{1} \mathrm{X}_{1}+\mathrm{c}_{2} \mathrm{X}_{2}+\ldots+\mathrm{c}_{\mathrm{n}} \mathrm{X}_{\mathrm{n}}=\frac{\left(X_{1}-\bar{X}\right) X_{1}}{\sum_{i=1}^{n}\left(X_{i}-\bar{X}\right)^{2}}+\frac{\left(X_{2}-\bar{X}\right) X_{2}}{\sum_{i=1}^{n}\left(X_{i}-\bar{X}\right)^{2}}+\ldots+\frac{\left(X_{n}-\bar{X}\right) X_{n}}{\sum_{i=1}^{n}\left(X_{i}-\bar{X}\right)^{2}}$

$$
\begin{aligned}
& =\frac{\sum_{i=1}^{n}\left(X_{i}-\bar{X}\right) X_{i}}{\sum_{i=1}^{n}\left(X_{i}-\bar{X}\right)^{2}} \\
= & \frac{\sum_{i=1}^{n} X_{i}^{2}-\bar{X} \cdot \sum_{i=1}^{n} X_{i}}{\sum_{i=1}^{n} X_{i}^{2}-2 \cdot \bar{X} \cdot \sum_{i=1}^{n} X_{i}+n \cdot \bar{X}^{2}} \\
= & \frac{\sum_{i=1}^{n} X_{i}^{2}-n \cdot \bar{X}^{2}}{\sum_{i=1}^{n} X_{i}^{2}-n \cdot \bar{X}^{2}}
\end{aligned}
$$

$c_{1} X_{1}+c_{2} X_{2}+\ldots+c_{n} X_{n}=1$

Jadi : $E(\hat{\beta})=\alpha(0)+\beta(1)=0+\beta=\beta$

Sehingga $\hat{\beta}$ merupakan penaksir tak bias bagi $\beta$. 


\section{Infínint ty}

\section{Distribusi dari Penaksir Tak Bias}

Dalam hal ini, penentuan distribusi dari penaksir $\hat{\beta}$ digunakan teknik fungsi pembangkit momen.

Dari uraian sebelumnya diperoleh :

$$
\hat{\beta}=c_{1} Y_{1}+c_{2} Y_{2}+\ldots+c_{n} Y_{n}
$$

Dengan : $c_{i}=\frac{\left(X_{i}-\bar{X}\right)}{\sum_{i=1}^{n}\left(X_{i}-\bar{X}\right)^{2}} \quad ; \quad i=1,2,3, \ldots, n$

Karena $Y_{1}, Y_{2}, \ldots, Y_{n}$ adalah sampel acak berukuran $n$ yang berasal dari populasi berdistribusi $N\left(\alpha+\beta X_{i} ; \sigma^{2}\right)$, maka fungsi pembangkit momen dari $Y_{i}$ adalah :

$$
\mathrm{M}_{\mathrm{Yi}}(\mathrm{t})=\exp \left[\left(\alpha+\beta \mathrm{X}_{\mathrm{i}}\right) \mathrm{t}+1 / 2 \sigma^{2} \mathrm{t}^{2}\right] ; t \in \mathfrak{R}
$$

Fungsi pembangkit momen dari $\hat{\beta}$ adalah:

$$
\begin{aligned}
M_{\hat{\beta}}(t) & =E[\exp (t \hat{\beta})] \\
& =\mathrm{E}\left[\exp \left\{\mathrm{t}\left(\mathrm{c}_{1} \mathrm{Y}_{1}+\mathrm{c}_{2} \mathrm{Y}_{2}+\ldots+\mathrm{c}_{\mathrm{n}} \mathrm{Y}_{\mathrm{n}}\right)\right\}\right] \\
& =\mathrm{E}\left[\exp \left(\mathrm{tc}_{1} \mathrm{Y}_{1}\right) \cdot \exp \left(\mathrm{tc}_{2} \mathrm{Y}_{2}\right) \ldots . \exp \left(\mathrm{tc}_{\mathrm{C}} \mathrm{Y}_{\mathrm{n}}\right)\right] \\
& =\mathrm{E}\left[\exp \left(\mathrm{tc}_{1} \mathrm{Y}_{1}\right)\right] \cdot \mathrm{E}\left[\exp \left(\mathrm{tc}_{2} \mathrm{Y}_{2}\right)\right] \ldots \cdot \mathrm{E}\left[\exp \left(\mathrm{tc}_{\mathrm{n}} \mathrm{Y}_{\mathrm{n}}\right)\right] \\
& =\mathrm{M}_{\mathrm{Y} 1}\left(\mathrm{c}_{1} \mathrm{t}\right) \cdot \mathrm{M}_{\mathrm{Y} 2}\left(\mathrm{c}_{2} \mathrm{t}\right) \ldots . . \mathrm{M}_{\mathrm{Yn}}\left(\mathrm{c}_{\mathrm{n}} \mathrm{t}\right) . \\
& =\left\{\exp \left[\left(\alpha+\beta \mathrm{X}_{1}\right) \mathrm{t}+1 / 2 \sigma^{2}\left(\mathrm{c}_{1} \mathrm{t}\right)^{2}\right]\right\} \cdot\left\{\exp \left[\left(\alpha+\beta \mathrm{X}_{2}\right) \mathrm{t}+1 / 2 \sigma^{2}\left(\mathrm{c}_{2} \mathrm{t}\right)^{2}\right]\right\} \\
& \ldots \ldots \cdot\left\{\exp \left[\left(\alpha+\beta \mathrm{X}_{\mathrm{n}}\right) \mathrm{t}+1 / 2 \sigma^{2}\left(\mathrm{c}_{\mathrm{n}} \mathrm{t}\right)^{2}\right]\right\} \\
& =\exp \left[\sum_{i=1}^{n}\left(\alpha+\beta X_{i}\right)\left(c_{i} t\right)+(1 / 2) \sigma^{2} t^{2} \cdot \sum_{i=1}^{n} c_{i}^{2}\right]
\end{aligned}
$$

Akan diuraikan bentuk yang ada pada pangkatnya.

- $\sum_{i=1}^{n}\left(\alpha+\beta X_{i}\right)\left(c_{i} t\right)=\sum_{i=1}^{n} \alpha \cdot c_{i} t+\sum_{i=1}^{n} \beta \cdot t \cdot c_{i} X_{i}$

$$
=\alpha \cdot t \sum_{i=1}^{n} c_{i}+\beta \cdot t \cdot \sum_{i=1}^{n} c_{i} X_{i}
$$

Karena dari uraian sebelumnya sudah diperoleh hasil bahwa $\sum_{i=1}^{n} c_{i}=0$ dan

$$
\begin{aligned}
& \sum_{i=1}^{n} c_{i} X_{i}=1, \text { maka: } \\
& \sum_{i=1}^{n}\left(\alpha+\beta X_{i}\right)\left(c_{i} t\right)=\alpha . t(0)+\beta . t(1)=0+\beta . t=\beta . t
\end{aligned}
$$


- $\quad \sum_{i=1}^{n} c_{i}^{2}=c_{1}^{2}+c_{2}^{2}+\ldots+c_{n}^{2}$

$$
\begin{gathered}
=\frac{\sum_{i=1}^{n}\left(X_{i}-\bar{X}\right)^{2}}{\left[\sum_{i=1}^{n}\left(X_{i}-\bar{X}\right)^{2}\right]^{2}} \\
\sum_{i=1}^{n} c_{i}^{2}=\frac{1}{\sum_{i=1}^{n}\left(X_{i}-\bar{X}\right)^{2}}
\end{gathered}
$$

Jadi : $M_{\hat{\beta}}(t)=\exp \left[\beta \cdot t+(1 / 2) \cdot \sigma^{2} t^{2} \cdot \frac{1}{\sum_{i=1}^{n}\left(X_{i}-\bar{X}\right)^{2}}\right]$

$$
=\exp \left[\beta \cdot t+(1 / 2) \cdot \frac{\sigma^{2}}{\sum_{i=1}^{n}\left(X_{i}-\bar{X}\right)^{2}} t^{2}\right]
$$

Ternyata bentuk di atas merupakan fungsi pembangkit momen dari distribusi normal dengan mean $=\beta$ dan varians $=\frac{\sigma^{2}}{\sum_{i=1}^{n}\left(X_{i}-\bar{X}\right)^{2}}$.

Sehingga $\hat{\beta}$ berdistribusi $N\left(\beta ; \frac{\sigma^{2}}{\sum_{i=1}^{n}\left(X_{i}-\bar{X}\right)^{2}}\right)$.

\section{Besaran Pivot}

Misalkan besaran pivotnya adalah:

$$
T=\frac{\hat{\beta}-\beta}{\sqrt{\frac{S_{e}^{2}}{\sum_{i=1}^{n}\left(X_{i}-\bar{X}\right)^{2}}}}
$$




\section{Distribusi dari Besaran Pivot}

Besaran pivot di atas bisa dituliskan kembali sbb:

$$
\begin{aligned}
& T=\frac{\hat{\beta}-\beta}{\sqrt{\frac{S_{e}^{2}}{\sum_{i=1}^{n}\left(X_{i}-\bar{X}\right)^{2}}}} \\
& T=\frac{\hat{\beta}-\beta}{\sqrt{\frac{\sigma^{2}}{\sum_{i=1}^{n}\left(X_{i}-\bar{X}\right)^{2}} \cdot \frac{(n-2) \cdot S_{e}^{2}}{\sigma^{2} \cdot(n-2)}}} \\
& T=\frac{\frac{\hat{\beta}-\beta}{\sqrt{\frac{\sigma^{2}}{\sum_{i=1}^{n}\left(X_{i}-\bar{X}\right)^{2}}}}}{\sqrt{\frac{(n-2) \cdot S_{e}^{2}}{\sigma^{2}} \cdot \frac{1}{n-2}}} \\
& T=\frac{W}{\sqrt{\frac{V}{n-2}}}
\end{aligned}
$$

Berikut ini akan diuraikan bentuk $\mathrm{W}$ dan $\mathrm{V}$.

$$
\text { - } W=\frac{\hat{\beta}-\beta}{\sqrt{\frac{\sigma^{2}}{\sum_{i=1}^{n}\left(X_{i}-\bar{X}\right)^{2}}}}
$$

Penentuan distribusi dari W akan digunakan teknik fungsi pembangkit momen.

Karena $\hat{\beta}$ berdistribusi $N\left(\beta ; \frac{\sigma^{2}}{\sum_{i=1}^{n}\left(X_{i}-\bar{X}\right)^{2}}\right)$, maka fungsi pembangkit momennya berbentuk: 


$$
M_{\hat{\beta}}(t)=\exp \left[\beta \cdot t+(1 / 2) \cdot \frac{\sigma^{2}}{\sum_{i=1}^{n}\left(X_{i}-\bar{X}\right)^{2}} t^{2}\right]
$$

Fungsi pembangkit momen dari $\mathrm{W}$ adalah: $\mathrm{M}_{\mathrm{W}}(\mathrm{t})=\mathrm{E}[\exp (\mathrm{tW})]$<smiles>[Y]C(=O)C(C)(C)C(C)(C)C</smiles>

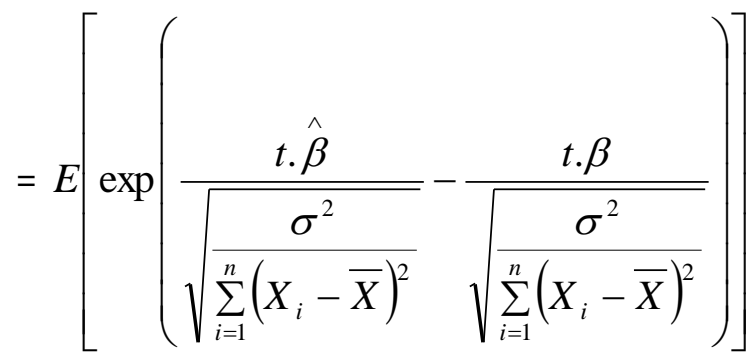

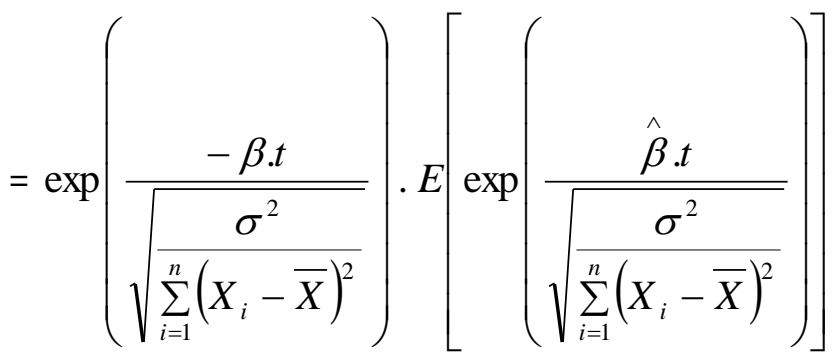

$$
=\exp \left(\frac{-\beta . t}{\sqrt{\frac{\sigma^{2}}{\sum_{i=1}^{n}\left(X_{i}-\bar{X}\right)^{2}}}}\right) \cdot M_{\hat{\beta}}\left[\left(\frac{t}{\sqrt{\frac{\sigma^{2}}{\sum_{i=1}^{n}\left(X_{i}-\bar{X}\right)^{2}}}}\right)\right]
$$




$$
=\exp \left(\frac{-\beta . t}{\sqrt{\frac{\sigma^{2}}{\sum_{i=1}^{n}\left(X_{i}-\bar{X}\right)^{2}}}}\right) \cdot \exp \left[\frac{\beta . t}{\sqrt{\frac{\sigma^{2}}{\sum_{i=1}^{n}\left(X_{i}-\bar{X}\right)^{2}}}}+(1 / 2) \cdot \frac{\sigma^{2}}{\sum_{i=1}^{n}\left(X_{i}-\bar{X}\right)^{2}} \cdot \frac{t^{2}}{\frac{\sigma^{2}}{\sum_{i=1}^{n}\left(X_{i}-\bar{X}\right)^{2}}}\right]
$$

$\mathrm{M}_{\mathrm{w}}(\mathrm{t})=\exp \left(1 / 2 \mathrm{t}^{2}\right)$

Ternyata bentuk di atas merupakan fungsi pembangkit momen dari distribusi normal baku.

Sehingga $\mathrm{W}$ berdistribusi $\mathrm{N}(0 ; 1)$.

- $V=\frac{(n-2) \cdot S_{e}^{2}}{\sigma^{2}}$

$$
\begin{aligned}
S_{e}^{2} & =\frac{1}{n-2} \sum_{i=1}^{n}\left(Y_{i}-\hat{Y}_{i}\right)^{2} \\
& =\frac{1}{n-2} \sum_{i=1}^{n}\left(Y_{i}-\hat{\alpha}-\hat{\beta} \cdot X_{i}\right)^{2}
\end{aligned}
$$

Dari uraian sebelumnya diperoleh $\hat{\alpha}=\bar{Y}-\hat{\beta} \cdot \bar{X}$ sehingga :

$$
\begin{aligned}
& S_{e}^{2}=\frac{1}{n-2} \sum_{i=1}^{n}\left(Y_{i}-\bar{Y}+\hat{\beta} \cdot \bar{X}-\hat{\beta} \cdot X_{i}\right)^{2} \\
& (n-2) \cdot S_{e}^{2}=\sum_{i=1}^{n}\left[Y_{i}-\bar{Y}-\hat{\beta}\left(X_{i}-\bar{X}\right)\right]^{2} \\
= & \sum_{i=1}^{n}\left[Y_{i}-\left(\hat{\beta}+\hat{\beta} \cdot X_{i}\right)+\left(\hat{\alpha}+\hat{\beta} \cdot X_{i}\right)-\bar{Y}-\hat{\beta}\left(X_{i}-\bar{X}\right)+(\alpha+\beta \cdot \bar{X})-(\alpha+\beta \cdot \bar{X})\right]^{2} \\
= & \sum_{i=1}^{n}\left[\left\{Y_{i}-\left(\alpha+\beta \cdot X_{i}\right)\right\}-\{\bar{Y}-(\alpha+\beta \cdot \bar{X})\}-\hat{\beta}\left(X_{i}-\bar{X}\right)+\beta\left(X_{i}-\bar{X}\right)\right]^{2} \\
& \sum_{i=1}^{n}\left[\left\{Y_{i}-\left(\alpha+\beta \cdot X_{i}\right)\right\}-\{\bar{Y}-(\alpha+\beta \cdot \bar{X})\}-(\hat{\beta}-\beta)\left(X_{i}-\bar{X}\right)\right]^{2}
\end{aligned}
$$




\section{Inffinit ity}

$$
\begin{aligned}
=\sum_{i=1}^{n}\left[Y_{i}\right. & \left.-\left(\alpha+\beta \cdot X_{i}\right)\right]^{2}+n \cdot[\bar{Y}-(\alpha+\beta \cdot \bar{X})]^{2}+(\hat{\beta}-\beta)^{2} \cdot \sum_{i=1}^{n}\left(X_{i}-\bar{X}\right)^{2} \\
& -2 \cdot \sum_{i=1}^{n}\left[Y_{i}-\left(\alpha+\beta \cdot X_{i}\right)\right][\bar{Y}-(\alpha+\beta \cdot \bar{X})] \\
& -2 \cdot \sum_{i=1}^{n}\left[Y_{i}-\left(\alpha+\beta \cdot X_{i}\right)\right]\left[(\hat{\beta}-\beta)\left(X_{i}-\bar{X}\right)\right] \\
& +2 \cdot \sum_{i=1}^{n}[\bar{Y}-(\alpha+\beta \cdot \bar{X})]\left[(\hat{\beta}-\beta)\left(X_{i}-\bar{X}\right)\right]
\end{aligned}
$$

Dengan :

$$
\text { (i) } \begin{aligned}
& \sum_{i=1}^{n}\left[Y_{i}-\left(\alpha+\beta \cdot X_{i}\right)\right][\bar{Y}-(\alpha+\beta \cdot \bar{X})] \\
= & \sum_{i=1}^{n} Y_{i} \cdot[\bar{Y}-(\alpha+\beta \cdot \bar{X})]-\sum_{i=1}^{n}\left(\alpha+\beta \cdot X_{i}\right)[\bar{Y}-(\alpha+\beta \cdot \bar{X})] \\
= & n \cdot \bar{Y} \cdot[\bar{Y}-(\alpha+\beta \cdot \bar{X})]-\left(n \cdot \alpha+\beta \cdot \sum_{i=1}^{n} X_{i}\right)[\bar{Y}-(\alpha+\beta \cdot \bar{X})] \\
= & n \cdot \bar{Y}^{2}-n \cdot \alpha \cdot \bar{Y}-n \cdot \beta \cdot \bar{X} \cdot \bar{Y}-n \cdot \alpha \cdot \bar{Y}-\beta \cdot \bar{Y} \cdot \sum_{i=1}^{n} X_{i}+n \cdot \alpha^{2}+\alpha \cdot \beta \cdot \sum_{i=1}^{n} X_{i} \\
& \quad+n \cdot \alpha \cdot \beta \cdot \bar{X}+\beta^{2} \cdot \bar{X} \cdot \sum_{i=1}^{n} X_{i} \\
= & n \cdot \bar{Y}^{2}-n \cdot \alpha \cdot \bar{Y}-n \cdot \beta \cdot \bar{X} \cdot \bar{Y}-n \cdot \alpha \cdot \bar{Y}-\beta \cdot \bar{Y} \cdot n \cdot \bar{X}+n \cdot \alpha^{2}+\alpha \cdot \beta \cdot n \cdot \bar{X} \\
& \quad+n \cdot \alpha \cdot \beta \cdot \bar{X}+\beta \beta^{2} \cdot \bar{X} \cdot n \cdot \bar{X} \\
= & n \cdot \bar{Y}^{2}-2 \cdot n \cdot \alpha \cdot \bar{Y}-2 \cdot n \cdot \beta \cdot \bar{X} \cdot \bar{Y}+n \cdot \alpha^{2}+2 \cdot \alpha \cdot \beta \cdot n \cdot \bar{X}+n \cdot \beta^{2} \cdot \bar{X}^{2} \\
= & n \cdot\left(\bar{Y}^{2}-2 \cdot \alpha \cdot \bar{Y}-2 \cdot \beta \cdot \bar{X} \cdot \bar{Y}+\alpha^{2}+2 \cdot \alpha \cdot \beta \cdot \bar{X}+\beta^{2} \cdot \bar{X}^{2}\right) \\
= & n \cdot\left[\bar{Y}^{2}-2 \cdot \bar{Y}(\alpha+\beta \cdot \bar{X})+(\alpha+\beta \cdot \bar{X})^{2}\right] \\
= & n \cdot[\bar{Y}-(\alpha+\beta \cdot \bar{X})]^{2}
\end{aligned}
$$

(ii) $\sum_{i=1}^{n}\left[Y_{i}-\left(\alpha+\beta . X_{i}\right)\right]\left[(\hat{\beta}-\beta)\left(X_{i}-\bar{X}\right)\right]$

$$
\begin{aligned}
& =(\hat{\beta}-\beta) \cdot \sum_{i=1}^{n}\left[Y_{i}-\left(\alpha+\beta \cdot X_{i}\right)\right]\left[X_{i}-\bar{X}\right] \\
& =(\hat{\beta}-\beta) \cdot\left[\sum_{i=1}^{n} X_{i} Y_{i}-\bar{X} \cdot \sum_{i=1}^{n} Y_{i}-\alpha \cdot \sum_{i=1}^{n}\left(X_{i}-\bar{X}\right)-\beta \cdot \sum_{i=1}^{n} X_{i}^{2}+\beta \cdot \bar{X} \cdot \sum_{i=1}^{n} X_{i}\right]
\end{aligned}
$$




$$
\begin{aligned}
& =(\hat{\beta}-\beta) \cdot\left[\sum_{i=1}^{n} X_{i} Y_{i}-\bar{X} \cdot n \cdot \bar{Y}-\alpha(0)-\beta \cdot \sum_{i=1}^{n} X_{i}^{2}+\beta \cdot n \cdot \bar{X}^{2}\right] \\
& =(\hat{\beta}-\beta) \cdot\left[\sum_{i=1}^{n} X_{i} Y_{i}-n \cdot \bar{X} \cdot \bar{Y}-\beta \cdot\left(\sum_{i=1}^{n} X_{i}^{2}-n \cdot \bar{X}^{2}\right)\right]
\end{aligned}
$$

Karena $\hat{\beta}=\frac{\sum_{i=1}^{n} X_{i} Y_{i}-n \cdot \bar{X} \cdot \bar{Y}}{\sum_{i=1}^{n} X_{i}^{2}-n \cdot \bar{X}^{2}}$, maka diperoleh :

$$
\begin{aligned}
& =(\hat{\beta}-\beta) \cdot\left[\hat{\beta}\left(\sum_{i=1}^{n} X_{i}^{2}-n \cdot \bar{X}^{2}\right)-\beta\left(\sum_{i=1}^{n} X_{i}^{2}-n \cdot \bar{X}^{2}\right)\right] \\
& =(\hat{\beta}-\beta) \cdot(\hat{\beta}-\beta)\left[\left(\sum_{i=1}^{n} X_{i}^{2}-n \cdot \bar{X}^{2}\right)\right] \\
& =(\hat{\beta}-\beta)^{2} \cdot \sum_{i=1}^{n}\left(X_{i}-\bar{X}\right)^{2}
\end{aligned}
$$

(iii) $\sum_{i=1}^{n}[\bar{Y}-(\alpha+\beta \cdot \bar{X})]\left[(\hat{\beta}-\beta)\left(X_{i}-\bar{X}\right)\right]$

$$
\begin{aligned}
& =[\bar{Y}-(\alpha+\beta \cdot \bar{X})](\hat{\beta}-\beta) \cdot \sum_{i=1}^{n}\left(X_{i}-\bar{X}\right) \\
& =[\bar{Y}-(\alpha+\beta \cdot \bar{X})](\hat{\beta}-\beta) .(0) \\
& =0
\end{aligned}
$$

Sehingga:

$$
\begin{aligned}
&(n-2) \cdot S_{e}^{2}=\sum_{i=1}^{n}\left[Y_{i}-\left(\alpha+\beta \cdot X_{i}\right)\right]^{2}+n \cdot[\bar{Y}-(\alpha+\beta \cdot \bar{X})]^{2}+(\hat{\beta}-\beta)^{2} \cdot \sum_{i=1}^{n}\left(X_{i}-\bar{X}\right)^{2} \\
&-2 \cdot n \cdot[\bar{Y}-(\alpha+\beta \cdot \bar{X})]^{2}-2 \cdot(\hat{\beta}-\beta)^{2} \cdot \sum_{i=1}^{n}\left(X_{i}-\bar{X}\right)^{2}+0 \\
&=\sum_{i=1}^{n}\left[Y_{i}-\left(\alpha+\beta \cdot X_{i}\right)\right]^{2}-n \cdot[\bar{Y}-(\alpha+\beta \cdot \bar{X})]^{2}-(\hat{\beta}-\beta)^{2} \cdot \sum_{i=1}^{n}\left(X_{i}-\bar{X}\right)^{2}
\end{aligned}
$$


Kedua ruas dibagi $\sigma^{2}$, sehingga diperoleh:

$$
\begin{aligned}
\frac{(n-2) \cdot S_{e}^{2}}{\sigma^{2}} & =\frac{\sum_{i=1}^{n}\left[Y_{i}-\left(\alpha+\beta \cdot X_{i}\right)\right]^{2}}{\sigma^{2}}-\frac{n \cdot[\bar{Y}-(\alpha+\beta \cdot \bar{X})]^{2}}{\sigma^{2}}-\frac{(\hat{\beta}-\beta)^{2} \cdot \sum_{i=1}^{n}\left(X_{i}-\bar{X}\right)^{2}}{\sigma^{2}} \\
= & \sum_{i=1}^{n}\left[\frac{Y_{i}-\left(\alpha+\beta \cdot X_{i}\right)}{\sigma}\right]^{2}-\left[\frac{\bar{Y}-(\alpha+\beta \cdot \bar{X}}{\frac{\sigma}{\sqrt{n}}}\right]^{2}-\left[\frac{\hat{\beta}-\beta}{\left.\frac{\sigma}{\sqrt{\sum_{i=1}^{n}\left(X_{i}-\bar{X}\right)^{2}}}\right]^{2}}\right.
\end{aligned}
$$

$\mathrm{A}=\mathrm{B}-\mathrm{C}-\mathrm{D}$

$\mathrm{B}=\mathrm{A}+\mathrm{C}+\mathrm{D}$

Fungsi pembangkit momen dari $\mathrm{B}$ adalah:

$$
\begin{aligned}
\mathrm{M}_{\mathrm{B}}(\mathrm{t}) & =\mathrm{E}[\exp (\mathrm{tB})] \\
& =\mathrm{E}[\exp \{\mathrm{t}(\mathrm{A}+\mathrm{C}+\mathrm{D})\}] \\
& =\mathrm{E}[\exp (\mathrm{tA}+\mathrm{tC}+\mathrm{tD})] \\
& =\mathrm{E}[\exp (\mathrm{tA}) \cdot \exp (\mathrm{tC}) \cdot \exp (\mathrm{tD})] \\
& =\mathrm{E}[\exp (\mathrm{tA})] \cdot \mathrm{E}[\exp (\mathrm{tC})] \cdot \mathrm{E}[\exp (\mathrm{tD})] \\
& =\mathrm{M}_{\mathrm{A}}(\mathrm{t}) \cdot \mathrm{M}_{\mathrm{C}}(\mathrm{t}) \cdot \mathrm{M}_{\mathrm{D}}(\mathrm{t})
\end{aligned}
$$

$$
M_{A}(t)=\frac{M_{B}(t)}{M_{C}(t) \cdot M_{D}(t)}
$$

Karena : a. Y berdistribusi $N\left(\alpha+\beta . X ; \sigma^{2}\right)$

$$
\begin{aligned}
& \frac{Y-(\alpha+\beta . X)}{\sigma} \text { berdistribusi } \mathrm{N}(0 ; 1) \\
& \left(\frac{Y-(\alpha+\beta . X)}{\sigma}\right)^{2} \text { berdistribusi } \chi^{2}(1) \\
& B=\sum_{i=1}^{n} B_{i}^{2}=\sum_{i=1}^{n}\left(\frac{Y_{i}-\left(\alpha+\beta . X_{i}\right)}{\sigma}\right)^{2} \text { berdistribusi } \chi^{2}(\mathrm{n}) \\
& \mathrm{M}_{\mathrm{B}}(\mathrm{t})=(1-2 \mathrm{t})(-1 / 2)(\mathrm{n}) ; \mathrm{t}<1 / 2
\end{aligned}
$$


b. $\bar{Y}$ berdistribusi $N\left(\alpha+\beta \cdot \bar{X} ; \frac{\sigma^{2}}{n}\right)$

$$
\begin{aligned}
& \frac{\bar{Y}-(\alpha+\beta \cdot \bar{X})}{\frac{\sigma}{\sqrt{n}}} \text { berdistribusi } \mathrm{N}(0 ; 1) \\
& C=\left(\frac{\bar{Y}-(\alpha+\beta \cdot \bar{X})}{\frac{\sigma}{\sqrt{n}}}\right)^{2} \text { berdistribusi } \chi^{2}(1) \\
& \mathrm{M}_{\mathrm{C}}(\mathrm{t})=(1-2 \mathrm{t})^{-1 / 2} ; \mathrm{t}<1 / 2
\end{aligned}
$$

c. $\hat{\beta}$ berdistribusi $N\left(\beta ; \frac{\sigma^{2}}{\sum_{i=1}^{n}\left(X_{i}-\bar{X}\right)^{2}}\right)$

$$
\begin{aligned}
& \frac{\hat{\beta}-\beta}{\frac{\sigma}{\sqrt{\sum_{i=1}^{n}\left(X_{i}-\bar{X}\right)^{2}}}} \text { berdistribusi } \mathrm{N}(0 ; 1) \\
& D=\left(\frac{\hat{\beta}-\beta}{\frac{\sigma}{\sqrt{\sum_{i=1}^{n}\left(X_{i}-\bar{X}\right)^{2}}}}\right)^{2} \text { berdistribusi } \chi^{2}(1)
\end{aligned}
$$

$$
M_{D}(t)=(1-2 t)^{-1 / 2} ; t<1 / 2
$$

Sehingga: $M_{A}(t)=\frac{(1-2 t)^{(-1 / 2) \cdot n}}{(1-2 t)^{-1 / 2} \cdot(1-2 t)^{-1 / 2}}=(1-2 t)^{(-1 / 2)(n-2)}$

Ternyata bentuk di atas merupakan fungsi pembangkit momen dari distribusi Chi-Kuadrat dengan derajat kebebasan $=\mathrm{n}-2$. 


\section{Infínint ty}

Sehingga bisa ditulis : $A=\frac{(n-2) \cdot S_{e}^{2}}{\sigma^{2}}$ berdistribusi $\chi^{2}(n-2)$.

Selanjutnya akan ditentukan distribusi dari $\mathrm{T}$ dengan menggunakan teknik transformasi peubah acak.

Misalkan $\mathrm{W}$ adalah peubah acak berdistribusi normal baku dan $\mathrm{V}$ adalah peubah acak berdistribusi Chi-Kuadrat dengan derajat kebebasan $\mathrm{dk}=\mathrm{r}=\mathrm{n}-2$. Kedua peubah acak $\mathrm{W}$ dan $\mathrm{V}$ saling bebas.

Jika peubah acak $T=\frac{W}{\sqrt{V / r}}$, maka akan ditentukan fungsi kepadatan peluang dari T.

Fungsi kepadatan peluang dari $\mathrm{W}$ adalah:

$$
f(w)=\frac{1}{\sqrt{2 \pi}} e^{-w^{2} / 2} ;-\infty<w<\infty
$$

Fungsi kepadatan peluang dari $\mathrm{V}$ adalah:

$$
\begin{aligned}
\mathrm{g}(\mathrm{v})= & \frac{1}{2^{r / 2} \cdot \Gamma(r / 2)} v^{(r / 2)-1} \cdot e^{-v / 2} ; 0<v<\infty \\
& =\quad 0 \quad ; \text { v lainnya. }
\end{aligned}
$$

Karena $\mathrm{W}$ dan $\mathrm{V}$ saling bebas, maka fungsi kepadatan peluang gabungannya adalah:

$\mathrm{h}(\mathrm{w}, \mathrm{v})=\mathrm{f}(\mathrm{w}) \cdot \mathrm{g}(\mathrm{v})$

$=\frac{1}{\sqrt{2 \pi}} e^{-w^{2} / 2} \cdot \frac{1}{2^{r / 2} \cdot \Gamma(r / 2)} v^{(r / 2)-1} \cdot e^{-v / 2} ;-\infty<w<\infty, 0<v<\infty$

$=0 ; \mathrm{w}, \mathrm{v}$ lainnya .

Karena transformasi peubah acak yang diketahui berbentuk $T=\frac{W}{\sqrt{V / r}}$, maka akan dimisalkan transformasi peubah acak keduanya adalah $\mathrm{U}=\mathrm{V}$.

Jadi transformasi peubah acaknya adalah $T=\frac{W}{\sqrt{V / r}}$ dan $\mathrm{U}=\mathrm{V}$. 
Hubungan antara nilai $\mathrm{w}$ dari $\mathrm{W}$ dan nilai $\mathrm{v}$ dari $\mathrm{V}$ dengan nilai $\mathrm{t}$ dari $\mathrm{T}$ dan nilai u dari U diberikan dengan:

$$
t=\frac{w}{\sqrt{v / r}} \text { dan } \mathrm{u}=\mathrm{v}
$$

Invers: $w=\frac{t \sqrt{u}}{\sqrt{r}}$ dan $\mathrm{v}=\mathrm{u}$

$$
\begin{aligned}
\text { Jacobiannya: } \mathrm{J} & =\left|\begin{array}{ll}
\frac{\partial w}{\partial t} & \frac{\partial w}{\partial u} \\
\frac{\partial v}{\partial t} & \frac{\partial v}{\partial u}
\end{array}\right|=\left|\begin{array}{cc}
\sqrt{u} / \sqrt{r} & t /(2 \sqrt{r u}) \\
0 & 1
\end{array}\right|=\frac{\sqrt{u}}{\sqrt{r}} \\
|J| & =\frac{\sqrt{u}}{\sqrt{r}}
\end{aligned}
$$

Fungsi kepadatan peluang gabungan dari T dan U adalah:

$$
\begin{aligned}
\mathrm{k}(\mathrm{t}, \mathrm{u})= & h\left(\frac{t \sqrt{u}}{\sqrt{r}}, u\right) \cdot|J| \\
\mathrm{k}(\mathrm{t}, \mathrm{u})= & \frac{1}{\sqrt{2 \pi} \cdot 2^{r / 2} \cdot \Gamma(r / 2)} u^{(e / 2)-1} \cdot \exp \left[\frac{-u}{2}\left(1+\frac{t^{2}}{r}\right)\right] \cdot \frac{\sqrt{u}}{\sqrt{r}} ;-\infty<\mathrm{t}<\infty, \\
& 0<\mathrm{u}<\infty \\
= & 0 ; \mathrm{t}, \mathrm{u} \text { lainnya. }
\end{aligned}
$$

Fungsi kepadatan peluang marginal dari T adalah:

$$
\begin{aligned}
k_{1}(t) & =\int_{-\infty}^{\infty} k(t, u) d u \\
& =\int_{0}^{\infty} \frac{1}{\sqrt{2 \pi} \cdot 2^{r / 2} \cdot \Gamma(r / 2)} u^{(r / 2)-1} \cdot \exp \left[\frac{-u}{2}\left(1+\frac{t^{2}}{r}\right)\right] \cdot \frac{\sqrt{u}}{\sqrt{r}} \mathrm{du} \\
& =\frac{1}{\sqrt{2 \pi} \cdot 2^{r / 2} \cdot \Gamma(r / 2)} \int_{0}^{\infty} u^{(r / 2)-(1 / 2)} \cdot \exp \left[\frac{-u}{2}\left(1+\frac{t^{2}}{r}\right)\right] \cdot \frac{\sqrt{u}}{\sqrt{r}} d u
\end{aligned}
$$




$$
\begin{aligned}
\text { Misalkan: } \frac{u}{2}\left(1+\frac{t^{2}}{r}\right)=y \\
u=\frac{2 y}{1+\frac{t^{2}}{r}} \\
d u=\frac{2}{1+\frac{t^{2}}{r}} d y
\end{aligned}
$$

Batas-Batas: Untuk $\mathrm{u}=0$, maka $\mathrm{y}=0$

Untuk $\mathrm{u}=\infty$, maka $\mathrm{y}=\infty$

$$
\begin{aligned}
& k_{1}(t)=\frac{1}{\sqrt{2 \pi} \cdot 2^{r / 2} \cdot \Gamma(r / 2)} \int_{0}^{\infty}\left(\frac{2 y}{\left.1+\frac{t^{2}}{r}\right)}\right)^{(r / 2)-(1 / 2)} \cdot \frac{2}{t^{2}} d y \\
&= \frac{2^{(r / 2)+(1 / 2)}}{\sqrt{2 \pi r} \cdot 2^{r / 2} \cdot \Gamma(r / 2) \cdot\left(1+\frac{t^{2}}{r}\right)^{(r+1) / 2}} \int_{0}^{\infty} y^{(r / 2)-(1 / 2)} \cdot e^{-y} d y \\
&= \frac{\Gamma\left(\frac{r+1}{2}\right)}{\sqrt{\pi r} \cdot \Gamma(r / 2) \cdot\left(1+\frac{t^{2}}{r}\right)^{(r+1) / 2}} \\
& \text { Maka: } k_{1}(t)=\frac{\Gamma\left(\frac{r+1}{2}\right)}{\sqrt{\pi r} \cdot \Gamma(r / 2) \cdot\left(1+\frac{t^{2}}{r}\right)^{(r+1) / 2}} ;-\infty<t<\infty \\
&
\end{aligned}
$$


Ternyata bentuk di atas merupakan fungsi kepadatan peluang dari distribusi $\mathrm{t}$ dengan derajat kebebasan $=r=n-2$.

\section{Besaran Pivot Disubstitusikan kedalam Bentuk Umum Taksiran Interval}

$$
P\left(-t_{(\alpha / 2) ;(n-2)}<\frac{\hat{\beta}-\beta}{\sqrt{\frac{S_{e}^{2}}{\sum_{i=1}^{n}\left(X_{i}-\bar{X}\right)^{2}}}}<t_{(\alpha / 2) ;(n-2)}\right)=1-\alpha
$$

6. Ubah Bentuk Taksiran Interval dalam Besaran Pivot Menjadi Bentuk Parameter

$$
P\left(\hat{\beta}-t_{(\alpha / 2) ;(n-2)} \cdot \sqrt{\frac{S_{e}^{2}}{\sum_{i=1}^{n}\left(X_{i}-\bar{X}\right)^{2}}}<\beta<\hat{\beta}+t_{(\alpha / 2) ;(n-2)} \cdot \sqrt{\frac{S_{e}^{2}}{\sum_{i=1}^{n}\left(X_{i}-\bar{X}\right)}}\right)=1-\alpha
$$

Sehingga taksiran interval dari $\beta$ dengan derajat keyakinan sebesar $(1-\alpha)$ berbentuk:

$$
\hat{\beta}-t_{(\alpha / 2) ;(n-2)} \cdot \sqrt{\frac{S_{e}^{2}}{\sum_{i=1}^{n}\left(X_{i}-\bar{X}\right)^{2}}}<\beta<\hat{\beta}+t_{(\alpha / 2) ;(n-2)} \cdot \sqrt{\frac{S_{e}^{2}}{\sum_{i=1}^{n}\left(X_{i}-\bar{X}\right)^{2}}}
$$

\section{Penutup}

Penentuan taksiran interval sebuah parameter dari sebuah distribusi dilakukan menggunakan metode besaran pivot, dengan langkah-langkah sbb:

1. Tentukan taksiran titik dari parameter itu. Dalam hal ini, tentunya dicari penaksir tak bias bagi parameter itu.

2. Tentukan distribusi dari penaksir tak bias itu (kalau diperlukan).

3. Tentukan besaran pivot, yaitu besaran yang mengandung penaksir dan parameter sedemikian hingga distribusinya tidak bergantung pada parameternya.

4. Tentukan distribusi dari besaran pivot. 
5. Besaran pivot itu disubstitusikan kedalam bentuk umum dari taksiran interval dengan derajat keyakinan sebesar $(1-\alpha)$, yaitu :

$$
\mathrm{P}(\mathrm{a}<\text { besaran pivot }<\mathrm{b})=1-\alpha
$$

6. Ubah bentuk dalam langkah kelima kedalam bentuk:

$$
\mathrm{P}(\mathrm{c}<\text { parameter }<\mathrm{d})=1-\alpha
$$

Taksiran interval dari $\beta$ dengan derajat keyakinan sebesar $(1-\alpha)$ berbentuk:

$$
\hat{\beta}-t_{(\alpha / 2) ;(n-2)} \cdot \sqrt{\frac{S_{e}^{2}}{\sum_{i=1}^{n}\left(X_{i}-\bar{X}\right)^{2}}}<\beta<\hat{\beta}_{(\alpha / 2) ;(n-2)} \cdot \sqrt{\frac{S_{e}^{2}}{\sum_{i=1}^{n}\left(X_{i}-\bar{X}\right)^{2}}}
$$

\section{Daftar Pustaka}

Clarke, G.M. \& Cooke, D. 1983. A Basic Course in Statistics. Second Edition. London : The English Language Book Society.

Freund, John E. \& Walpole, Ronald E. 1980. Mathematical Statistics. Third Edition. New York : Prentice-Hall, Inc, Englewood.

Guttman, Irwin; Wilks, S.S; dan Hunter, J.Stuart. 1982. Introductory Engineering Statistics. Third Edition. New York : John Willey \& Sons.

Herrhyanto, Nar. 2003. Statistika Matematis Lanjutan. Bandung : Pustaka Setia.

Herrhyanto, Nar \& Gantini, Tuti. 2009. Pengantar Statistika Matematis. Bandung : Penerbit Yrama-Widya.

Herrhyanto, Nar. 2010. Bank Soal Teori Statistika Matematik dan Penyelesaiannya. Bandung : Penerbit Yrama Widya.

Hogg, Robert V. dan Tanis, Elliot A. 1977. Probability and Statistical Inference. New York : Macmillan Publishing Co., Inc. 